\title{
A rat model of early stage osteonecrosis induced by glucocorticoids
}

\author{
Mohammad Amin Kerachian ${ }^{1,2}$, Edward J Harvey ${ }^{3}$, Denis Cournoyer ${ }^{1,4,5}$, Terry Y Chow ${ }^{5}$, Ayoub Nahal ${ }^{6}$ and \\ Chantal Séguin ${ }^{4,5^{*}}$
}

\begin{abstract}
Background: Glucocorticoid (GC)-induced osteonecrosis (ON) is an important complication of medical therapy. The exact pathomechanisms of ON has not been clearly elucidated. There is a need for a reproducible animal model that better approximates the clinical scenario.

Methods: To determine the genetic susceptibility of rats to develop GC-induced femoral head ON, we evaluated 5 different inbred strains of rats (Spontaneous Hypertensive Rat, Wistar Kyoto, Wistar Furth, SASCO Fisher and Lewis). Prednisone pellets (dosage of $1.82-2.56 \mathrm{mg} / \mathrm{kg} /$ day) were implanted subcutaneously for 90 . After 90 days, the femurs were resected and examined histologically and radiographically. Pathological and histological examination was performed. Hematoxylin and eosin ( $H$ \& E) staining was used to delineate the femoral head osteonecrosis lesions as well as abnormalities of articular cartilage and growth plate.
\end{abstract}

Results: The greatest differences in H \& E staining were seen in the Wistar Kyoto and Wistar Furth groups. In these groups 4 out of 5 and 3 out of 5, respectively, steroid-induced rats revealed growth plate disruption with acellular areas. The TUNEL apoptosis staining assay for apoptosis revealed that 4 out of 5 of Wistar Kyoto rats, 5 out of 5 of Wistar Furth, 2 out of 4 of surviving Lewis and 2 out of 2 of the surviving spontaneous hypertensive rats had apoptotic osteocytes in trabeculae, whereas none of the Fisher rats showed apoptotic osteocytes.

Conclusions: We postulate that Wistar Kyoto, Wistar Furth and spontaneous hypertensive rats may be strains of rats more susceptible to develop ON of the femoral head while Fisher rats were the most resistant.

\section{Background}

Glucocorticoids (GCs) are widely prescribed in cases of rheumatoid arthritis, asthma, systemic lupus erythematosus, cancer, organ transplantation and many other medical conditions. The therapeutic use of GCs has been accompanied by marked side effects, especially with the long-term usage of this drug. The adverse effect of GCs on bone has been recognized for more than 60 years [1-3]. The bone effect is characterized by decreased bone formation and in situ death of isolated segments of bone which may be associated with osteonecrosis $(\mathrm{ON})$ particularly important clinically for the femoral head. ON in the femoral head gradually progresses to fracture of the subchondral bone, collapse of the surface and hip arthritis. Although ON has been

\footnotetext{
* Correspondence: chantal.seguin@muhc.mcgill.ca

${ }^{4}$ Department of Medicine, Division of Haematology, McGill University Health Center (MUHC), 1650 Cedar Avenue, Montreal, QC H3G 1A4, Canada Full list of author information is available at the end of the article
}

linked to a variety of conditions, GC usage remains the predisposing factor most commonly associated with the development of non-traumatic ON. There is considerable interest in identifying which patients are at highest risk for $\mathrm{ON}$, with the long-term goal of modifying regimens to decrease the risk of adverse effects of therapy. Despite the strong association of GC administration with $\mathrm{ON}$, the role of potential underlying risk factors such as hyperlipidemia, thrombophilia, and hyperfibrinolysis in the circulatory system remain unclear $[2,4]$. It has been clearly established that among patients receiving a specific dose of GC, only an unpredictable subset will develop ON. This underscores the existence of individual variability in the action of GCs and the potential presence of additional mechanisms and/or risk factors such as a genetic predisposition. On the other hand, studying the clinical pathology of $\mathrm{ON}$ in the early disease stage (before radiographic findings) is extremely difficult in human subjects. Thus, animal experiments

\section{() Biomed Central}


are needed to elucidate the pathophysiology of the disorder. Having a suitable animal model would allow for the systemic evaluation of host-related (ie. genetic variations) as well as acquired (ie. treatment-related) risk factors. GC-induced $\mathrm{ON}$ has been induced in rabbit models [5-8], bipedal animals (e.g., chickens, emus) $[9,10]$ and recently, in BALB/cJ mice [11]. GC-induced $\mathrm{ON}$ has been described in mature Japonese white rabbits (Kbs-JW) [5,8] but the genome of rabbit has only been incompletely sequenced, thus limiting the usefulness of that model for the identification of genes affecting the risk of developing ON. The biped models are difficult to interpret in the context of bone healing as we do not have a full grasp of avian bone healing. Although a mouse model of GC-induced ON is interesting, the very small diameter of the femoral head of mice limits the application of numerous experiments and monitoring techniques. It is currently impossible to read an MRI or radiograph from a mouse with the goal of differentiating a normal hip from a hip with ON changes. A rat model would allow easier radiographic interpretation, allow facile surgical interventions, allow existing small animal facilities to be used as well as be in an animal where the genetics of healing is much better understood. To date, there has been no rat model of GC-induced $\mathrm{ON}$ unless it has been combined with a surgical procedure [12] or in combination with immune responses stimuli [13]. These blood interruption studies do not faithfully model the more prevalent non-traumatic ON. In this study, our goal was to establish a rat model of GC-induced $\mathrm{ON}$ by screening different strains of rats in order to uncover those whose constitutive phenotype might predispose to the development of ON.

\section{Methods}

\section{Maintenance and experimental animals}

In this pilot study, female retired breeder (aged 6-8 months) Fisher, Lewis, Spontaneous Hypertensive, Wistar Kyoto, and Wistar Furth rats (6 of each strain) were obtained from Charles River Laboratories (Pointe-Claire, QC, Canada). The rats were tagged and housed in plastic cages ( 2 animals per cage) under standard laboratory conditions with a 12-hour dark/12-hour light cycle, a constant temperature of $20^{\circ} \mathrm{C}$, and humidity of $48 \%$. Food and water were provided ad libitum with a standard rodent diet. The weight of the rats were followed before and after the implant of a prednisone pellet for the first 4 consecutive days, then every week until the end of the experiment. All experiments were conducted under an animal protocol (Protocol No. 4935) approved by the McGill Animal Care Department, Montreal, Canada.

\section{Glucocorticoid administration}

Slow-release prednisone pellets (Innovative Research of America, Sarasota, Florid, USA) were implanted subcutaneously in 5 inbred rats composing each group (Fisher, Lewis, Spontaneous Hypertensive, Wistar Kyoto and Wistar Furth). Each pellet was implanted underneath the skin on the lateral side of the neck by surgically making an incision and developing a pocket about $2 \mathrm{~cm}$ beyond the incision site. The pellet was placed in the pocket and the incision was sutured. Based on the manufacturer's instructions the pellet releases a constant dose of the drug subcutaneously. The average dose release from the pellet was equivalent to $1.82-2.56 \mathrm{mg} /$ $\mathrm{kg} /$ day (mean: 2.26, SD: 0.19) for a period of 90 days. This dosage is an equivalent dosage to humans that commonly causes $\mathrm{ON}$ changes. Thus, each group had 5 GC-induced rats along with 1 control rat in each group not treated with prednisone (the control rat did not receive a placebo pellet).

\section{Histological Examination}

The rats were sacrificed with an overdose of ketamine/ xylazine following 90 days of the experiment. Tissue samples were obtained from the femoral head. Bone samples were fixed in $10 \%$ neutral buffered formalin overnight, then decalcified in $4 \%$ ethylenediamine tetraacetic acid (pH 7.2) (Sigma-Aldrich, St. Louis, MO, USA). The specimens were processed routinely and embedded in paraffin. Tissue sections were cut parasagitally with a rotary microtome to obtain 4 to 5 microns thickness, stained with hematoxylin and eosin (H \& E) and evaluated by light microscopy.

Tissue samples were analyzed in a blinded fashion by an experienced bone pathologist (AN). GC-induced ON was diagnosed based on bone and growth plate changes. The histological findings of an established ON were defined as dead trabeculae exhibiting empty lacunae with or without appositional bone formation [14]. Occasional empty lacunae possibly created by sectioning through the edge of a lacunae was not considered as a sign for $\mathrm{ON}$. The growth plate changes were considered as thinning, discontinuity pattern and disruption of articular cartilage alignment or growth plate alignment.

Tissue sections were also examined according to the criteria of Arlet et al. namely presence of degeneration, necrosis, and disappearance of marrow cells as well as the nuclear disappearance and hypochromasia of trabecular osteocytes as early signs of ON [15]. Early signs of $\mathrm{ON}$ was also considered when apoptosis occurred in the osteocytes and osteoblasts. Positivity for apoptosis was defined by the authors as 2 to 3 apoptotic osteocytes and/or osteoblasts considered as one plus, between 3 to 6 as two plus and more than 6 as three plus recognized 
in a high magnification field $(\times 200)$. The experiments were performed in triplicate.

\section{Measurement of apoptosis in undecalcified bone section} We used terminal deoxynucleotidyl transferase dUTP nick end labeling (TUNEL assay) to detect DNA fragmentation by labeling the terminal end of nucleic acids. In Situ Cell Death Detection Kit was obtained from Roche (Germany). TUNEL assay on paraffin-embedded tissue sections was performed as recommended by the manufacturer. Briefly, after deparaffinization and permeabilization of the tissue sections with proteinase $\mathrm{K}$, the slides were incubated with the TUNEL reaction mixture containing TUNEL-Enzyme solution and TUNELLabel solution for 1 hour at $37^{\circ} \mathrm{C}$ inside a humidified chamber. After washing steps, samples were analyzed under a fluorescence microscope (in a drop of $1 \times$ PBS). The excitation wavelength ranged between 450-500 nm whereas the detection wavelength ranged between 515$565 \mathrm{~nm}$ (green). DNase I-treated tissue section was used as a positive control. Negative controls for the study constituted of sample slides processed using the same procedure but only treated with TUNEL-Label solution.

\section{Faxitron X-ray}

Based on the histological results Faxitron $\mathrm{x}$-ray analysis was performed initially on a group of Wistar Kyoto rats (5 rats, 10 femoral head samples) (Model MX-20). Previous work has shown that radiographic changes were a late finding in steroid induced $\mathrm{ON}$ in the rat model used. We performed the Faxitron radiographs on this group to ensure there were no significant changes despite changes on histology.

\section{Statistical Analysis}

Comparison between groups was made with Fisher's Exact test. Significant differences were considered at $\mathrm{P}$ values less than 0.05 .

\section{Results}

We observed a high mortality rate in some strains of rats after prednisone implantation. Interestingly, the Lewis and spontaneous hypertensive strains of rats seemed to be at highest risk (mortality rate was 1 out of 5 and 3 out of 5 , respectively and no mortality for other strains). There was an overall mortality rate of $16 \%$ among the steroid-treated rats in our pilot study related to the development of GC-induced hyperglycemia in these "older" rats (a two to three times fold increase compare to control rats).

Growth plate changes were observed in Wistar Kyoto and Wistar Furth rats (Figure 1). In these groups 4 out of 5 and 3 out of 5 of steroid-induced rats revealed growth plate disruption with acellular areas, respectively.
Osteocyte necrosis and empty lacunae were not detected in any samples. TUNEL assay for apoptosis revealed that 4/5 of Wistar Kyoto, 5/5 of Wistar Furth, 2/4 of Lewis and 2/2 Spontaneous Hypertensive rats had apoptotic osteocytes in trabeculae, whereas none of the Fisher rats showed apoptotic osteocytes (Table 1, Figure 2). In the Lewis group, apoptosis of osteocytes and osteoblasts without any degeneration of the growth plate was observed. Overall, the most apoptosis rate was in spontaneous hypertensive rats $(+++)$ and then Wistar Furth $(++)$ and Wistar Kyoto $(++)$. The apoptosis level in Lewis and Fisher rats was $(+)$ and zero, respectively. Bone marrow and chondrocyte apoptotic cells were seen in all strains of rats, even the control rats as expected. There were no signs of inflammation and necrosis, such as hyperemia, round cell infiltration, or lipid cyst formation. Plain $\mathrm{x}$-rays obtained from Faxitron analysis did not reveal any significant anomaly in the initial group of Wystar Kyoto rats. Often diminished bone density was noticed in rats exposed to glucocorticoid. The radiographs were not performed in the other groups because of the lack of changes. If there had been changes in the initial group the other strains would have been tested. This finding also confirmed that plain $\mathrm{x}$-rays are not a suitable method to diagnose early stages of $\mathrm{ON}$ in rats.

\section{Discussion}

A strain dependant genetic predisposition may be responsible for the high mortality rate observed in some strains of rats after prednisone implantation. Recognition of this complication of hyperglycemia has been reported in young rats [16] and seems to be important when choosing a model for ON. It would make these two strains less desirable for this usage.

The rat growth plate is present throughout the lifecycle. This may confound the findings of $\mathrm{ON}$ for late stage $\mathrm{ON}$ in that reparative changes will overcome the initiating stimulus- particularly in traumatic vascular interruption studies. For this GC study, the high dosages of steroid given will result in early ongoing changes in the rat femoral head despite reparative processes from the growth plate. Because of this a rodent model is possible for early ON.

Growth plate disruption was observed particularly in Wistar Kyoto and Wister Furth strains. This was observed in early stages of the disease- before radiographic change was evident. Other studies using blood supply interruption (ischemic model) have also shown growth plate changes. Trueta and Amato used animal models and showed that the blood supply to the cartilage of the growth plate of the femoral head originates from the epiphyseal vessels [17], while the metaphysis is supplied by metaphyseal vessels and nutrient arteries coming from the medullary cavity. Mechanical damage 

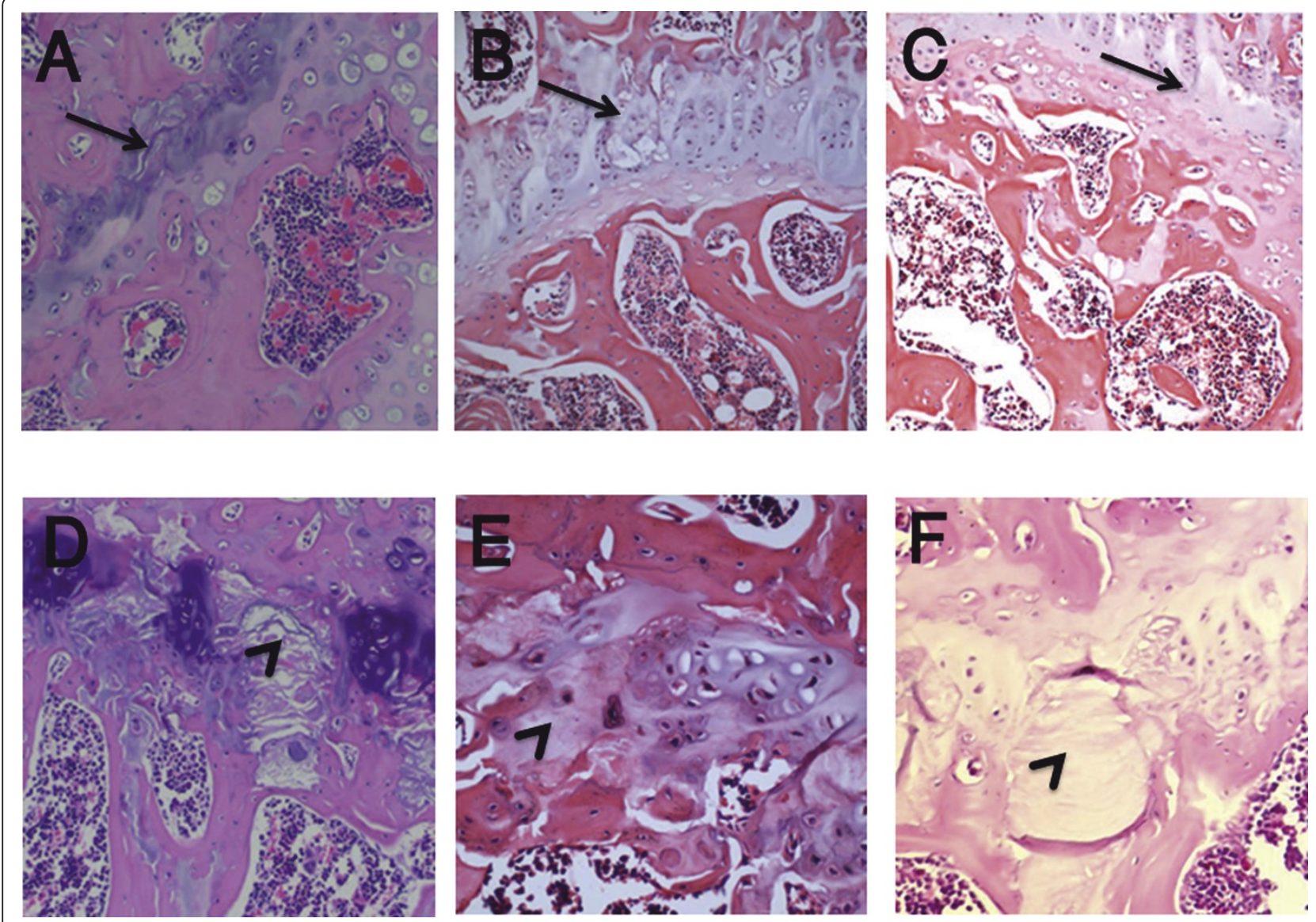

Figure 1 Photomicrographs showing histological findings in steroid-induced rat models. H \& E staining in a control rat (A) versus glucocorticoid-induced rats of all groups (B: Lewis, C: Fisher, D: Wistar Kyoto, E: Wistar Furth and F: Spontaneous Hypertensive rats). Normal growth plate and its discontinuity pattern are shown by arrows and arrowheads, respectively. Magnification $\times 200$.

to the metaphyseal arteries leads to destruction of the growth plate and, eventually, a physeal bridge [18]. It is possible that thrombosis in the metaphyseal arteries reported in $\mathrm{ON}$ of the femoral head could cause injury and disruption of the growth plate with areas lacking normal cells. Sato et al. have also shown that apoptosis tended to occur in early stages of ON. In their rat study of ischemic ON, apoptosis occurred 12 hours after the mechanical insult, whereas no evidence of apoptosis

Table 1 Apoptosis of the femoral head of GC-induced inbred rats from 5 different strains (WKY: Wistar Kyoto, WF: Wistar Furth, SHR: Spontaneous Hypertensive rat,).

\begin{tabular}{cccc}
\hline Strain & Bone Marrow & Osteocyte & Chondrocyte \\
\hline Lewis & $4 / 4$ & $2 / 4^{*}$ & $4 / 4$ \\
Fischer & $5 / 5$ & $0 / 5^{*}$ & $5 / 5$ \\
WKY & $5 / 5$ & $4 / 5^{*}$ & $5 / 5$ \\
WF & $5 / 5$ & $5 / 5^{*}$ & $5 / 5$ \\
SHR & $2 / 2$ & $2 / 2^{*}$ & $2 / 2$ \\
\hline
\end{tabular}

*Fisher's Exact Test ( $P$ value $=0.0039)$. remained after 96 hours, at which time only empty lacunae were detected [19]. They postulated that the mechanism of cell death involved in ischemic $\mathrm{ON}$ was apoptosis as indicated by DNA fragmentation and the presence of apoptosis bodies in osteocytes $[11,20]$. In the present study, apoptosis of chrondrocytes were not only detected in GC-induced rats but also in control rats indicating that apoptosis of chondrocytes is not characteristic of ON but probably more indicative of normal bone turnover if observed in small amounts. Other studies have shown that apoptosis of osteocytes and osteoblasts is an important process in developing $\mathrm{ON}$, especially in the early stages of ON [21]. Kabata and his colleagues demonstrated extensive osteocyte apoptosis in a rabbit model of GC-induced ON [22]. Shibahara et al. also reported the presence of a large number of apoptotic osteocytes around necrotic areas [23]. In the present study, we observed that apoptosis occurred at the level of osteocytes, osteoblasts, and bone marrow cells in the early stages of GC-induced $\mathrm{ON}$ lesions in three strains of inbred rats: Wistar Kyoto, 

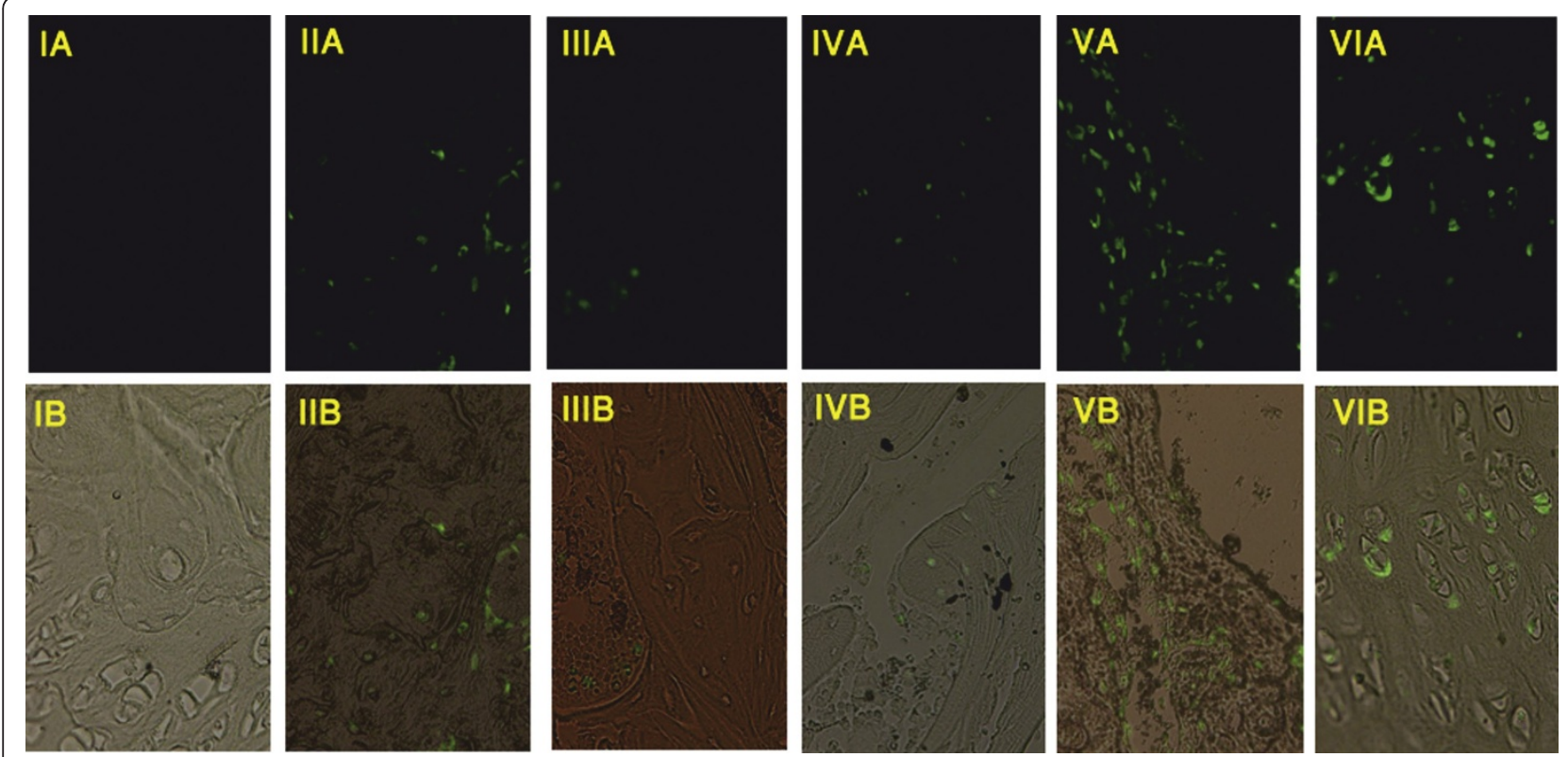

Figure 2 Photomicrographs showing apoptosis of osteocytes as a marker of early osteonecrosis of the femoral head. TUNEL staining apoptosis assay analyzed by (A): florescence microscope, (B): superimposed florescence and optical view on femoral head in II: Lewis, III: Fisher, IV: Wistar Kyoto, V: Wistar Furth and VI: Spontaneous Hypertensive rats induced with steroids for 3 months versus a control sample (I). The nucleus of apoptotic cells are shown in green. Magnification $\times 200$.

Wistar Furth and Spontaneous Hypertensive. Apoptosis has been shown to play an important role in maintaining haematopoietic stem cells (HSC) homeostasis in in vivo. Thus, apoptosis of the HSC could occur as part of the normal physiology in bone marrow cells [24]. Previously, it has been shown that apoptosis could happen in bone marrow of control rats [25]. Apoptosis could result from a direct effect of GCs on the bone cells or could be secondary to the dysfunction/activation of other cells such as the femoral head endothelial cells [26]. Fisher rats were resistant to the development of osteocyte apoptosis in response to GC induction. Given the observed inter-strain variability of susceptibility to the development of GC-induced ON lesions, it is probable that genetic factors are involved in ON developing in response to GCs. To date different genetic variations and mutations accounting for $\mathrm{ON}$ have been reported. A 4G/4G mutation of the plasminogen activator inhibitor-1 gene [27], a G- > A transition in exon 50 (p.G1170S) of collagen type II (COL2A1) [28] and a promoter polymorphisms of the vascular endothelial growth factor (VEGF) gene [29] were reported to be correlated with the occurrence of $\mathrm{ON}$ of the femoral head. Identifying the genetic factors may prove relevant to the human disorder and facilitate the identification of individuals at increased risk of developing ON.

\section{Conclusions}

Based on these findings, Wistar Kyoto, Wistar Furth and Spontaneously Hypertensive rats were the most susceptible strains to develop GC-induced $\mathrm{ON}$ of the femoral head. The Spontaneously Hypertensive rats had a high mortality rate that is unacceptable for a study model. Fisher rats were resistant to the development of $\mathrm{ON}$ at the GC dosage used, based on the absence of osteocyte apoptosis in early stages of the disease process. Although several other investigations have reported $\mathrm{ON}$ in rats following the administration of GCs, our rat model has shown early stage disease regardless of additional adjuvants such as surgery [12] or immune response stimuli $[13,30]$ as previously reported. It is possible that extended exposure to GCs could establish the histological criteria of the later stages of $\mathrm{ON}$.

\section{Acknowledgements}

This work has been supported by the Montreal General Hospital Foundation (C.S.), by the generous research award from Mr John D. Miller (C.S.) and support from FRSQ Chercheur-Boursier Clinicien Senior (E.J.H.).

\section{Author details}

'Department of Human Genetics, McGill University Health Center (MUHC), 1650 Cedar Avenue, Montreal, QC H3G 1A4, Canada. 2Department of Medical Genetics, Mashhad University of Medical Sciences (MUMS), Azadi Square, Mashhad, 917794-8564, Iran. ${ }^{3}$ Division of Orthopaedic Surgery, McGill University Health Center (MUHC), 1650 Cedar Avenue, Montreal, QC H3G 1A4, Canada. ${ }^{4}$ Department of Medicine, Division of Haematology, McGill 
University Health Center (MUHC), 1650 Cedar Avenue, Montreal, QC H3G 1A4, Canada. ${ }^{5}$ Department of Oncology, McGill University Health Center (MUHC), 1650 Cedar Avenue, Montreal, QC H3G 1A4, Canada. ${ }^{6}$ Department of Pathology, McGill University Health Center (MUHC), 1650 Cedar Avenue, Montreal, QC H3G 1A4, Canada.

\section{Authors' contributions}

All authors participated in the study. MAK made a major contribution to the writing of the manuscript's first draft, and conducted the experiments involved in the study. CS made a major contribution to the design of the study, data interpretation and scientific revision of the manuscript. DC, EJH and TYC made equal contributions to data interpretation and scientific revision of the manuscript. EJH made a major contribution to the editing and grammar of the manuscript. AN made major contributions to the histological experiments involved in the study. All authors participated in the manuscript preparation and revision. All authors read and approved the final manuscript.

\section{Competing interests}

The authors declare that they have no competing interests.

Received: 11 March 2011 Accepted: 21 December 2011

Published: 21 December 2011

\section{References}

1. Cushing $\mathrm{H}$ : The basophil adenomas of the pituitary body and their clinical manifestations (pituitary basophilism). 1932. Obes Res 1994, 2:486-508.

2. Kerachian MA, Séguin C, Harvey EJ: Glucocorticoids In Osteonecrosis of the Femoral Head: A New Understanding of the Mechanisms of Action. J Steroid Biochem Mol Biol 2009, 114:121-8.

3. Yao W, Cheng Z, Busse C, Pham A, Nakamura MC, Lane NE: Glucocorticoid excess in mice results in early activation of osteoclastogenesis and adipogenesis and prolonged suppression of osteogenesis: a longitudinal study of gene expression in bone tissue from glucocorticoid-treated mice. Arthritis Rheum 2008, 58:1674-86.

4. Jones LC, Mont MA, Le TB, Petri M, Hungerford DS, Wang P, Glueck CJ: Procoagulants and osteonecrosis. J Rheumatol 2003, 30:783-91.

5. Zhang G, Sheng $H, H e Y X$, et al: Continuous occurrence of both insufficient neovascularization and elevated vascular permeability in rabbit proximal femur during inadequate repair of steroid-associated osteonecrotic lesions. Arthritis Rheum 2009, 60:2966-77.

6. Zhang G, Wang XL, Sheng $H$, et al: Constitutional flavonoids derived from Epimedium dose-dependently reduce incidence of steroid-associated osteonecrosis not via direct action by themselves on potential cellular targets. PLoS One 2009, 4:e6419.

7. Zhang G, Qin L, Sheng H, Yeung KW, et al: Epimedium-derived phytoestrogen exert beneficial effect on preventing steroid-associated osteonecrosis in rabbits with inhibition of both thrombosis and lipiddeposition. Bone 2007, 40:685-92.

8. Sheng HH, Zhang GG, Cheung WH, et al: Elevated adipogenesis of marrow mesenchymal stem cells during early steroid-associated osteonecrosis development. J Ortho Surg Res 2007, 2:15-22.

9. Wang GJ, Cui Q, Balian G: The Nicolas Andry award. The pathogenesis and prevention of steroid-induced osteonecrosis. Clin Orthop Relat Res 2000, 370:295-310

10. Conzemius MG, Brown TD, Zhang Y, Robinson RA: A new animal model of femoral head osteonecrosis: one that progresses to human-like mechanical failure. J Orthop Res 2002, 20:303-309.

11. Yang L, Boyd K, Kaste SC, Kamdem KL, Rahija RJ, Relling MV: A mouse model for glucocorticoid-induced osteonecrosis: effect of a steroid holiday. J Orthop Res 2009, 27:169-175.

12. Chen $Y$, Huang $K$, Lang F, Huang $Y$, Huang $H$, Huang $H$, et al: Experimental study on cheng zai wan for treatment of necrosis of the femoral head. $J$ Tradit Chin Med 2003, 23:292-298.

13. Okazaki S, Nishitani Y, Nagoya S, et al: Femoral head osteonecrosis can be caused by disruption of the systemic immune response via the toll-like receptor 4 signalling pathway. Rheumatology (Oxford) 2009, 48:227-32.

14. Wada M, Kumagai $K$, Murata M, Yamashita Y, Shindo H: Warfarin reduces the incidence of osteonecrosis of the femoral head in spontaneously hypertensive rats. J Orthop Sci 2004, 9:585-590.
15. Arlet J: A traumatic necrosis of the femoral head: general report. In Bone circulation and vascularization in normal and pathological conditions. Edited by: Schoutens A, Arlet J, Gardeniers JWM, Hughes SPF. New York: Plenum; 1993:235-240.

16. Rafacho A, Cestari TM, Taboga SR, Boschero AC, Bosqueiro JR: High doses of dexamethasone induce increased beta-cell proliferation in pancreatic rat islets. Am J Physiol Endocrinol Metab 2009, 296:E681-9.

17. Trueta J, Amato VP: The vascular contribution to osteogenesis. III. Changes in the growth cartilage caused by experimentally induced ischaemia. J Bone Joint Surg Br 1960, 42-B:571-587.

18. Nyska M, Shabat S, Long PH, Howard C, Ezov N, Levin-Harrus T, et al: Disseminated thrombosis-induced growth plate necrosis in rat: a unique model for growth plate arrest. J Pediatr Orthop 2005, 25:346-350.

19. Sato M, Sugano N, Ohzono K, Nomura S, Kitamura Y, Tsukamoto Y, et al: Apoptosis and expression of stress protein (ORP150, HO1) during development of ischaemic osteonecrosis in the rat. J Bone Joint Surg Br 2001, 83:751-759.

20. Weinstein RS, Manolagas SC: Apoptosis in glucocorticoid-induced bone disease. Curr Opin Endocrinol Diabetes 2008, 12:219-223.

21. Jones LC, Hungerford DS: The pathogenesis of osteonecrosis. Instr Course Lect 2007, 56:179-196.

22. Kabata T, Kubo T, Matsumoto T, Nishino M, Tomita K, Katsuda S, et al: Apoptotic cell death in steroid induced osteonecrosis: an experimental study in rabbits. J Rheumatol 2000, 27:2166-2171.

23. Shibahara M, Nishida K, Asahara H, Yoshikawa T, Mitani S, Kondo Y, Inoue H: Increased osteocyte apoptosis during the development of femoral head osteonecrosis in spontaneously hypertensive rats. Acta Med Okayama 2000, 54:67-74.

24. Domen J: The role of apoptosis in regulating hematopoietic stem cell numbers. Apoptosis 2001, 6:239-52.

25. Matsouka P, Mylonas P, Papandoniou E, Dimitropoulou I, Floratou K, Alexandridis T, Kardamakis D: Abdominal radiation initiates apoptotic mechanism in rat femur bone marrow cells in vivo that is reversed by IGF-1 administration. J Radiat Res (Tokyo) 2008, 49:41-7.

26. Kerachian MA, Harvey EJ, Cournoyer D, Chow TY, Séguin C: Avascular Necrosis of the Femoral Head: Vascular Hypotheses. Endothelium 2006, 13:237-244.

27. Glueck CJ, Fontaine RN, Gruppo R, Stroop D, Sieve-Smith L, Tracy T, Wang P: The plasminogen activator inhibitor-1 gene, hypofibrinolysis, and osteonecrosis. Clin Orthop Relat Res 1999, 336:133-46.

28. Liu YF, Chen WM, Lin YF, Yang RC, Lin MW, et al: Type II collagen gene variants and inherited osteonecrosis of the femoral head. $N$ Engl J Med 2005, 352:2294-2301

29. Kim TH, Hong JM, Lee JY, Oh B, Park EK, Lee CK, Bae SC, Kim SY: Promoterpolymorphisms of the vascular endothelial growth factor gene is associated withan osteonecrosis of the femoral head in the Korean population. Osteoarthritis Cartilage 2008, 16:287-291.

30. Okazaki S, Nishitani Y, Nagoya S, Kaya M, Yamashita T, Matsumoto H: Femoral head osteonecrosis can be caused by disruption of the systemic immune response via the toll-like receptor 4 signalling pathway. Rheumatology (Oxford) 2009, 48:227-32.

doi:10.1186/1749-799X-6-62

Cite this article as: Kerachian et al:: A rat model of early stage

osteonecrosis induced by glucocorticoids. Journal of Orthopaedic Surgery and Research 2011 6:62. 